\title{
One Way of Bringing Final Year Computer Science Student World to the World of Children with Cerebral Palsy: A Case Study
}

\author{
Isabel M. Gómez ${ }^{1}$, Rafael Cabrera ${ }^{1}$, Juan Ojeda ${ }^{1}$, Pablo García ${ }^{1}$, \\ Alberto J. Molina ${ }^{1}$, Octavio Rivera ${ }^{1}$, and A. Mariano Esteban ${ }^{2}$ \\ ${ }^{1}$ Electronic Technology Department, Universidad de Sevilla, Spain \\ \{igomez, almolina\}@us.es, \{rcabrera, octavio\} adte.us.es, \\ \{juanojeda8,roll_cagesh\}@hotmail.com \\ ${ }^{2}$ Guadaltel, S.A. Seville, Spain \\ amer@guadaltel.es \\ http://matrix.dte.us.es/grupotais
}

\begin{abstract}
In this paper, a learning project is explained which is being carried out at the school of computer science at the University of Seville. The aim is that students receive knowledge of assistive technologies when in fact there is no this discipline in our curricula. So the best way, it is programming final studies projects in this field. We want to make the projects have a real application and can solve difficulties that children with Cerebral Palsy have in their daily activities in the school.
\end{abstract}

Keywords: serious games, trainig in assistive technologies, access device.

\section{The R \& D or Application Idea}

In this paper, a learning project is explained which is being carried out at the school of computer science at the University of Seville. The idea is that students who are completing their studies design applications that will contribute to improve the daily school activities of children with motor and cognitive problems. Training and age of these students are good ingredients for achieving this goal. On the one hand technical training in programming and electronics is ideal for application design. On the other hand students are of the generation of video games which they love and have great experience as players. Additionally, this project supplies assistive technologies Knowledge that is lacking in their studies. These applications will be games but there are other possibilities such as interactive stories or learning applications. The student must design them according to a preset goal. The properly access device must also be selected or designed according to the child's physical capabilities.

\section{State of the Art}

Games can be a very important tool in the lives of children with disabilities. If a high degree of involvement is reached, they can increase children capabilities, improve 
motor skills, promote sensory processing, and develop perception, social, emotional and language abilities. It is essential to have appropriate adaptations in all facets of the game use.

New technologies have positively influenced the access of children with great difficulty to games. In many cases helping them overcome their difficulties in a much more optimal way than ordinary rehabilitation therapy. In [1,2] the use of virtual reality systems making child perform exercises in proper way is shown. With these systems, the child shows greater interest, involvement and fun. In [3], virtual reality environments are described simply as a good alternative for leisure time. This will improve self-esteem and help feel the subject more capable. A very thorough assessment of the scope of these games on children with neuromotor impairments is done in [4].

There is a concept called "Serious Game" that is related to the use of games for purposes beyond entertainment. Serious games that are used for learning should have the power to captivate the player for a specific purpose such as acquiring new knowledge or skills [5]. The European Union funded project called Game On Extra Time (GOET), aims to prepare students with sensory and intellectual disabilities for real life situations. A series of serious games are used. The description of such games and their evaluation can be found in [6]. On the other hand [7] is a study of the factors that contribute to literacy for children with cerebral palsy. It is highlighted the importance of educational software to achieve this goal.

The proper choice of access devices helps the user feel comfortable with the application, reducing fatigue and frustration caused by the inability to manipulate a system. Thus the main objectives that are intended: learning, communication and rehabilitation; can be achieved more easily. It is important to consider that solutions and sensors must be adapted to the subjects. The use of switches is very common, but our conclusion after visiting the school analyzing capacities and behavior of children is that accelerometers can be a better alternative. An example of using accelerometers to design an access device can be found in [8]. Another possibility can be sounds acquired through sound card input.

\section{The Methodology Used}

The aim of the project described in this paper is based on a visit to a school on a normal day, in which interviews were held with staff who care for children. This allows us to establish some working lines related solving of specific needs in different environments: learning in classroom, stories in the library, stimulation in multi sensory classroom and games in the school playground.

A structured system with the aim that all students work with the same pattern is proposed. Different modules that compound the system are independent and thus the system is flexible because modules of the same level can be exchanged and have the possibility to be extended. Figure 1 shows system structure. The acquisition module performs the data reading coming from a user device. This device can be a switch, an accelerometer, a microphone or any hardware adapted to user needs. It is important to 
discern a voluntary action in data set. The calibration module must be implemented with each user before he starts using the application module. It should be based on classifiers. It is configured to distinguish between the captured data, those used for the application control. Once the user begins using the application, the event detection module detects when the user has generated the control event. Once this detection is produced it will generate a command to the module of the application in order to perform the action corresponding to that event.

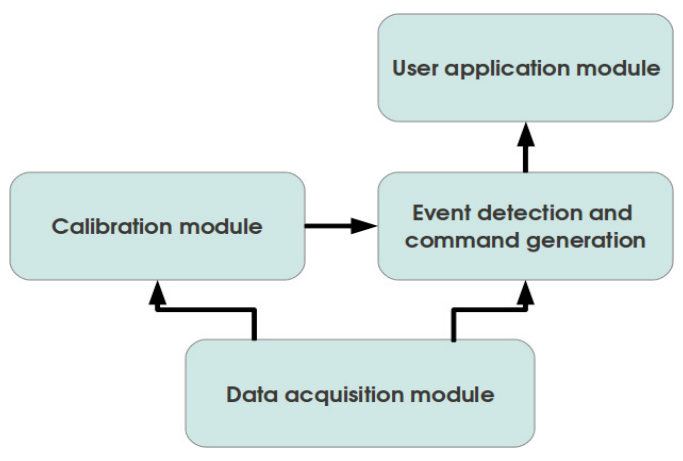

Fig. 1. System Structure

Application module consists of games, interactive stories and learning software. The objective should be to encourage capabilities that this type of user has in a basic level: postural control, action_ reaction, learning curricular materials of different natures and levels. Handling and contents must be designed to respond adequately to a particular user profile. For example, in the case of a person with severe motor difficulties, management will be discrete (on-off), so a single event achieves big changes in application operation. Development should be done in phases and not launch to the next one until the previous one has been assessed by the staff there. Development will be done for both personal computers and mobile devices.

Event detection and command generation module transmits commands to application module in the form of a mouse event. So the interface of applications can only works with this kind of event. This is a constraint in order to make modules designed by different students be interchangeable.

The following programming languages for the realization of this work: $\mathrm{C}++, \mathrm{C} \mathrm{\#}$ and Java have been evaluated. Between them $\mathrm{C \#}$ has been chosen for several reasons: it is easy to use; it has a function library dedicated to game design; it has a framework called XNA Game Studio to facilitate the development of games; games developed for a personal computer can be compiled for XBOX and and make them available on Xbox Live [9].The following section describes how to realize this methodology to a specific case. 


\section{Case Study: Racing Car Game}

In figure 2 main menu of the game entitled CARRERAS US (RACING University of Seville) is shown. This objective of this game is to promote action-reaction capability in children.

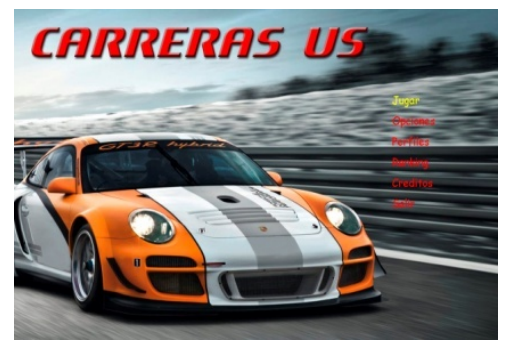

Fig. 2. Game Main Menu

Next it describes how different modules of the methodology are implemented. Overall system architecture is shown in figure 3.

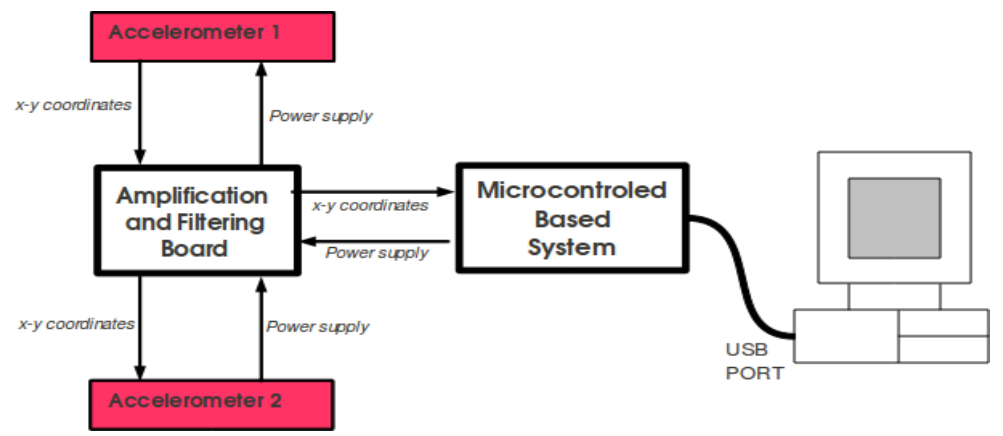

Fig. 3. System Architecture

1. Acquisition module. It is based on accelerometers. Two ADXL322 are used for this purpose. This device is a low cost, low power, complete dual-axis accelerometer with signal conditioned voltage outputs, which is all on a single monolithic IC. The product measures acceleration with a full-scale range of $\pm 2 \mathrm{~g}$. Amplification and filtering board is connected to the accelerometer; the aim of this board is to quickly eliminate undesired movements and it allows the system to work with a reduced angle range what increments system usability. The microcontroller-based system reads the accelerometer $\mathrm{x}-\mathrm{y}$ coordinates and communicates that acceleration to a PC. Another function of this system is to perform digital signal processing. In order to increment the system effectiveness, we have programmed a moving average filter. Arduino has been used for implementing this system. Arduino is an open-source electronics prototyping platform based on flexible, easy-to-use hardware and software [10]. It is based on the Atmel Atmega328 microcontroller. 
2. Calibration module. This game has several modes: continuous, discrete, one or two players. In discrete mode, a voluntary movement of the children is selected to generate the event. In the calibration module this is done establishing a threshold in order to distinguish this movement among involuntary ones. This is a simple procedure that will not be valid in all cases, it being necessary to establish more complex classifiers. In continuous mode, users' movements are adjusted to screen dimensions. In this module, user profiles can be considered in order to avoid calibration once it has already been done.

3. Event detection and command generation module. This is once the system calibration is done. If an event is detected, this module translates it to mouse events, cursor movement or click, depending on game mode.

4. Application module. This module has easy graphical interface according to the explanation given by that school psychologist. In figure 4, game screens are shown; a car appears in movement but in determined instant, the situation changes and a reaction is required by the user. If the reaction is good and in time, user wins points, if not, the car crashes. Depending on the game mode, the action required in users is different: in discrete, only the voluntary movement used in calibration time; in continuous, a more precise movement is required being careful not to pass road limits.

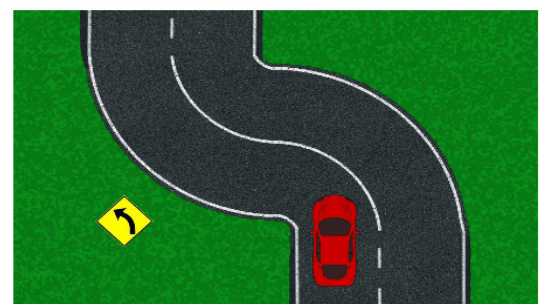

a. When a curve is shown, this requires an user action to take it properly

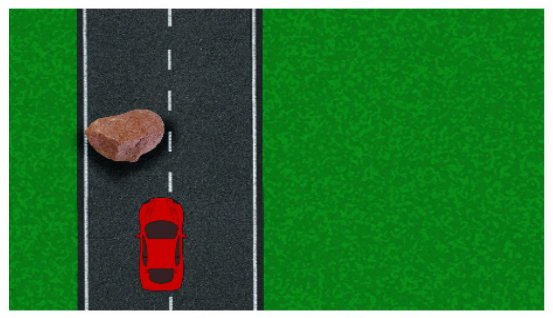

c. A stone requires user reaction too.

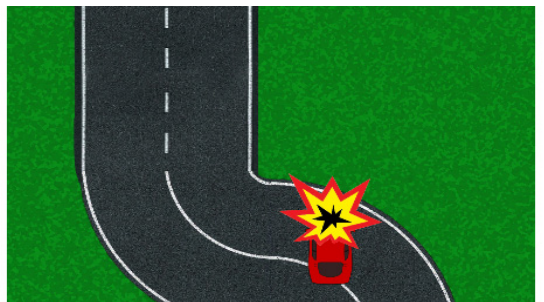

b.If the user doesn't react when the curve appears, the car doesn't take it properly. So It is crash

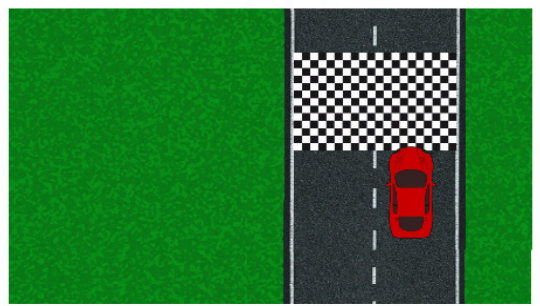

d. Game over. Points are given to user

Fig. 4. Game Screens

\section{$5 \quad$ Results and Discussion}

\subsection{Application}

This game is for children with low motor and cognitive capabilities. For this reason, several phases have been considered each one with different goals. In a first stage of 
using the game, it is better one player and one event mode. For this case, we only need an adequate reaction in the user when a stimulus appears in the game screen. In a second stage, when the user has experience, it is possible two events mode, the user must decide between move the car to the left or right. In this case the cognitive capability needed is increased. Two players' mode allows to develop cooperation between children, they learn to respect game turns and work together to obtain the punctuation. Continuous mode allows increasing motor capabilities.

Sound effects like music or car's crash noise have been added in order to stimulate participation and avoid bore. Car speed can be configured too in order to adapt users' needs, expert level and capabilities.

\subsection{Access Device}

Discrete Mode. It is important distinguish between voluntary and involuntary movements. In this case, it is assumed that involuntary movements are slower which means lower values in accelerometer coordinates. For this reason, the following algorithm is used:

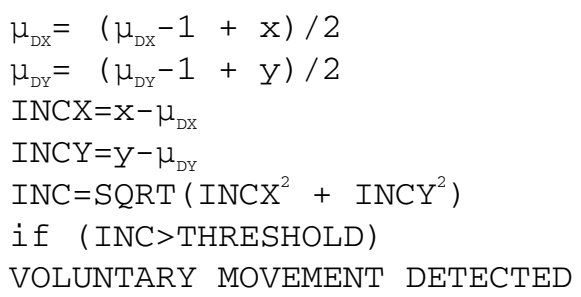

$\mu_{\mathrm{DX}}$ and $\mu_{\mathrm{DY}}$ are the mean values of the coordinate $\mathrm{x}$ and $\mathrm{y}$ measured with the accelerometer. $\mu_{\mathrm{D}-1}$ is the previous mean value.

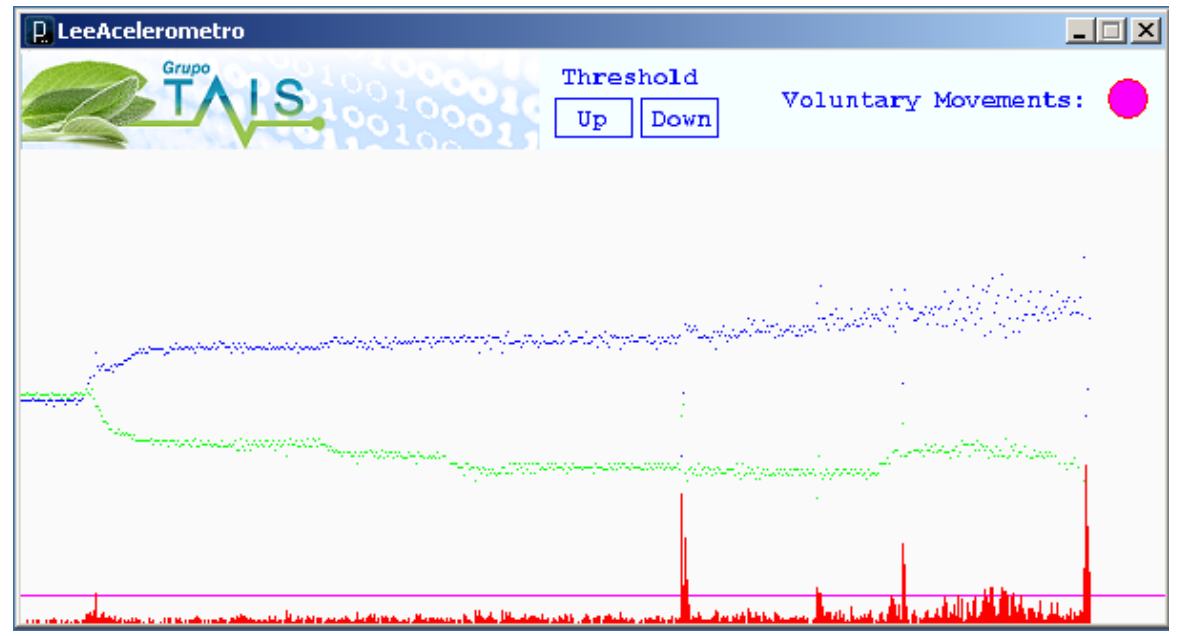

Fig. 5. High part of the image: Accelerometer captured data. Low part: threshold and INC are represented. Threshold can be tuned. 
This way of event generation is more flexible and comfortable than using a switch device because it can be adjusted more easily to users' capabilities and preferences.

Continuous Mode. Only needs adjustment to screen resolution according to users' movement

\section{Conclusion and Planned Activitities}

In this paper an educational experience that takes place in the computer science degree at the University of Seville has been described. The goal is to connect two groups (computer science students and children with disabilities), so that both can broaden their point perspectives and benefit. These are recent happenings so a complete evaluation of it is not yet available. Up to now, students implied in it are motivated and working with interest.

There are several groups of students working on different applications with several aims; one of these has been described. To finish the students' work properly, the school psychologist must evaluated it and finally test it with the children.

Acknowledgments. This project has been carried out within the framework of a research program: (p08-TIC-3631) - Multimodal Wireless interface funded by the Regional Government of Andalusia.

\section{References}

1. Bryanton, C., Bossé, J., Brien, M., Mclean, J., Mccormick, A., Sveistrup, H.: Feasibility, Motivation, and Selective Motor Control: Virtual Reality Compared to Conventional Home Exercise in Children with Cerebral Palsy. Cyberpsychology \& Behavior 9(2) (2006)

2. Reid, D.T.: Benefits of a Virtual Play Rehabilitation Environment for Children with Cerebral Palsy on Perceptions of Self-Efficacy: a Pilot Study. Pediatric Rehabilitation 5(3), 141-148 (2002)

3. Weiss, P.L., Bialik, P., Kizony, R.: Virtual Reality Provides Leisure Time Opportunities for Young Adults with Physical and Intellectual Disabilities. Cyberpsychology \& Behavior 6(3) (2003)

4. Levac, D., Rivard, L., Missiuna, C.: Defining the Active Ingredients of Interactive Computer Play Interventions for Children with Neuromotor Impairments: a Scoping Review. Research in Developmental Disabilities 33 (2012)

5. Susi, T., Johannesson, M., Backlund, P.: Serious Games - An Overview Technical Report HS-IKI-TR-07-001 (Key: citeulike:1137029 (February 5, 2007)

6. Sik Lanyi, C., Brown, D.J., Standen, P., Lewis, J., Butkute, V.: User Interface Evaluation of Serious Games for Students with Intellectual Disability. In: Miesenberger, K., Klaus, J., Zagler, W., Karshmer, A. (eds.) ICCHP 2010, Part I. LNCS, vol. 6179, pp. 227-234. Springer, Heidelberg (2010)

7. Peeters, M., de Moor, J., Verhoeven, L.: Emergent Literacy Activities, Instructional Adaptations and School Absence of Children with Cerebral Palsy in Special Education. Research in Developmental Disabilities 32 (2011)

8. Nakazawa, N., Yamada, K., Matsui, T., Itoh, I.: Development of Welfare SupportEquipment for Personal Computer Operation with Head Tilting and Breathing. In: IECON Thirty-First Annual Conference of the IEEE Industrial Electronics Society (2005)

9. Official webpage XBOX, http: //www. xbox. com

10. Arduino, http://www . arduino.cc/ 